\title{
Prevalence and predictors of verbal aggression in a secure mental health service: use of the HCR-20
}

Cevher Gunenc, Laura E. O'Shea and Geoffrey L. Dickens

This is the peer reviewed version of the following article:

Gunenc, C., O'Shea, L.E. and Dickens, G.L. 2015. Prevalence and predictors of verbal aggression in a secure mental health service: use of the HCR-20. International Journal of Mental Health Nursing. 24(4): pp.314-323. doi: 10.1111io/inm.12130

which has been published in final form at http://dx.doi.org/10.1111/ inm.12130. This article may be used for non-commercial purposes in accordance with Wiley Terms and Conditions for Self-Archiving. 


\section{Prevalence and predictors of verbal aggression in a secure mental health service:Use of the HCR-20}

Cevher Gunenc, 1 Laura E. O'Shea1,2 and Geoffrey L. Dickens1,3 1St. Andrew's Academic Department, St. Andrew's Hospital, Northampton, and 2 Institute of Psychiatry, King's

College London, London, and 3School of Health and Social Sciences, Abertay University, Dundee, UK 


\begin{abstract}
Despite evidence about the negative effects of verbal aggression in mental health wards, there is little research about its prevalence, or about the factors that predict the behaviour among inpatients. This study aimed to determine the prevalence of verbal aggression in a secure mental health service, and to examine the relationship of verbal aggression with risk factors for aggression in the risk assessment tool HCR-20 in order to establish whether, and with which factors, the behaviour can be predicted. Verbal aggression was measured using the Overt Aggression Scale (OAS) over a three-month period across a heterogeneous patient group $(N=613)$. Over half the patients $(n=341 ; 56 \%)$ engaged in 1,594 incidents of verbal aggression. The HCR-20 total, clinical, and risk management subscale scores predicted verbal aggression, though effect sizes were not large. Item-outcome analysis revealed that impulsivity, negative attitudes, and noncompliance with medication were the best predictors of verbal aggression and, therefore, should be targeted for intervention. There are key synergies between factors predicting verbal aggression and the core mental health nursing role. Nurses, therefore, are in a prime position to develop and implement interventions that may reduce verbal aggression in mental health inpatients.
\end{abstract}

Keywords: Inpatient, language, aggression, nurses, risk-management 


\section{Introduction}

Physical aggression and violence in psychiatric settings has been explored extensively but there has been relatively little investigation into the prevalence, causes, and effects of verbal aggression (Stewart \& Bowers, 2013). Verbal aggression is a common event on psychiatric wards, more so even than physical aggression (Arnetz \& Arnetz, 2001; Kay et al., 1988; Rippon, 2000) with staff being the most frequent victims (Stewart \& Bowers, 2013). As many as $70-90 \%$ of staff in psychiatric settings have experienced verbal aggression (Duxbury, 2002). Verbal aggression can be as psychologically damaging as physical assault; Flannery Jr. et al. (1995) reported victims of verbal threats to have symptoms similar to Post Traumatic Stress Disorder, and to experience negative effects for as long as 10 days after the incident. Stone et al. (2010; p. 532) reported that nurses experienced effects similar to those of physical assault, for example 'feeling sick and bruised'. Further, O'Connell et al. (2000) found a higher percentage of nurses reported feelings of anxiety, frustration, guilt, and helplessness after experiencing verbal aggression compared with physical aggression. Verbal aggression can also bear on nurse-patient relationships (Needham et al., 2005). Nurses may feel resentment towards the verbally aggressive patient, and, thus, persistent verbal aggression may result in the patient receiving a lower quality of care (Arnetz \& Arnetz, 2001).

Despite the prevalence, and potentially severe consequences of verbal aggression, much of the available research has explored only severe types of the behaviour such as threats of violence (Flannery Jr. et al., 1995), or examined the behaviour of relatively homogeneous samples such as males diagnosed with schizophrenia (Werner et al., 1983). Most studies of the correlates of verbal aggression have examined only demographic factors such as age and gender (Grassi et al., 2001), and have not attempted to explore a wider range of dynamic clinical and attitudinal predictors. Therefore, research is needed to investigate the 
full range of verbal aggression, and to identify both relatively static and potentially modifiable dynamic risk factors. Further, we need to accurately predict which patients are at increased risk for engaging in verbal aggression in order to implement effective preventive strategies and interventions.

The HCR-20 (Webster et al., 1997) is a widely used tool comprising 20 historical and dynamic risk factors to assist clinicians in the assessment of violence risk in psychiatric patients. It has also been found to predict behaviours such as self-harm (O'Shea, Picchioni, et al., 2014a) and inappropriate sexual behaviour (O'Shea, Thaker, et al., 2014) in mental health wards. It would therefore be pragmatic, and of potential utility, to ascertain whether the HCR-20 has predictive efficacy for verbal aggression. Further, the analysis of the predictive ability of the individual risk factors and subscales within the HCR-20 would facilitate identification of those factors which best predict verbal aggression, and would suggest potentially fruitful intervention targets. The aim of this study, therefore, was to describe the prevalence of the full range of verbal aggression in a diverse inpatient sample, and to identify the associated static and dynamic risk factors. A further aim was to investigate whether verbal aggression and physical aggression towards others overlap in an inpatient setting as this has implications for the feasibility of using the HCR-20 to predict both outcomes.

\section{Method}

Setting and participants

St. Andrew's is a UK charity specialising in the treatment and rehabilitation of people with mental disorder; almost all are detained under civil and forensic sections of the Mental Health Act (1983, amended 2007). The Charity provides specialist inpatient services for men, women, and older-adults with mental illness, neuropsychiatric disorders, intellectual disability, personality disorder, and developmental disorders, in a range of medium secure, 
low secure, and locked units. To be eligible for inclusion, patients must have been present in the service between $1^{\text {st }}$ September 2010 and $31^{\text {st }}$ March 2011, and assessed with the HCR-20. Patients were excluded if they did not remain in the service for at least three months after assessment.

Design and procedure

The study followed the same pseudo-prospective design as O'Shea, Picchioni, et al. (2014a), where anonymised demographic/clinical patient information and data from routine HCR-20 assessments, carried out by qualified psychologists or supervised assistant psychologists, were obtained. Descriptions of incidents of verbal aggression occurring in the three-month period after assessment were also retrieved from electronic records.

\section{Measures}

Violence risk assessment

The HCR-20 is a 20-item tool used to predict violence in people diagnosed with mental disorder. The HCR-20 comprises three subscales: Historical (10 items; H10), Clinical (5 items; C5), and Risk Management (5 items; R5). Each item is scored on a 3-point scale: No or 0 (absent), Possible or 1 (possibly present), and Yes or 2 (definitely present). Clinicians then consider the ratings together with case-specific factors to form an overall summary judgement (SJ) of low, moderate, or high risk for future violence. In this study, the SJ refers to the risk of future violence causing serious physical harm. The risk-management items are rated twice in clinical practice, considering the patients in institutional (In) or community (Out) settings (R5). Only 'In' scores were used as the purpose of this research was to measure inpatient verbal aggression. Item 7 (psychopathy) was omitted from the H10 scale due to missing scores for the majority of patients as specialist training is required to administer the 
Psychopathy Checklist Revised (PCL-R; Hare, 1991). The HCR-20 has been reported to have equivalent predictive validity without the psychopathy item (Guy et al., 2010). HCR-20 scores were prorated to account for missing values in accordance with instructions in the manual (Webster et al., 1997). Version 2 (Webster et al., 1997) was used at the time of data collection, though version 3 is now available (Douglas et al., 2013).

\section{Demographic and clinical data}

Demographic information, including patient's gender, age at assessment, time between admission and assessment, ethnicity, ICD-10 diagnosis (World Health Organization, 1992), security level on the ward, and legal status was retrieved from patient records. Individual consent from patients was not required to access this information as data was coded anonymously with identifying details removed; therefore, it cannot be traced back to the patient.

\section{Outcome measure}

Incidents of verbal aggression and physical aggression towards other people recorded during the three months following assessment were extracted from progress notes and coded using the Overt Aggression Scale (OAS; Yudofsky et al., 1986); inter-rater reliability (Kappa $.81-1.0)$ was in the excellent range. The OAS requires verbal aggression to be rated on a fourpoint criterion referenced severity scale from 1 (makes loud noises, shouts angrily), through 2 (yells mild personal insults [e.g. "You're stupid!”]), 3 (curses viciously, uses foul language in anger, makes moderate threats to others or self), and 4 (makes clear threats of violence toward others or self [“I'm going to kill you"] or requests to help control self). However, only three outcomes were measured in this study: 'mild verbal aggression', which included criteria 1 and 2 of the OAS, 'severe verbal aggression', including criteria 3 and 4, and 'any verbal aggression' (all criteria 1 to 4 ). 


\section{Data analysis}

All analyses were performed using SPSS version 18 for Windows (SPSS, 2009). Descriptive statistics were used to describe the sample and outcome base rates. Independent $t$ tests and one-way ANOVAs were used to examine differences in HCR-20 scores as a function of risk level assigned by the SJ, and between those who engaged in each of the three outcomes and those who did not. Further, Pearson's chi squared tests were used to examine differences in prevalence of verbal aggression between the three levels of risk and between different groups within the sample e.g., gender and age; additionally, overlap between engagement in physical and verbal aggression was analysed. Area Under the Curve (AUC) values from receiver operating characteristic (ROC) analyses were used to determine the predictive validity of the HCR-20, and its individual items for the three categories of verbal aggression derived from OAS scores: mild, severe, and any verbal aggression. Each item was analysed separately to account for missing scores on some items. AUC values measure predictive validity and range from 0 (perfect negative prediction) to 1 (perfect positive prediction), with .5 indicating zero predictive validity (Dernevik et al., 2002). The threshold for a large effect size is typically considered to be .75 (Doyle \& Dolan, 2002); in this context, this indicates that a randomly selected verbally aggressive patient will score higher than a randomly selected non-verbally aggressive patient on the HCR-20 in $75 \%$ of cases.

\section{Results}

Participants

Of 692 eligible patients, 52 were excluded due to missing information, and 27 because they did not remain in the service for three months following HCR-20 assessment, leaving a final sample of $N=613$. Two thirds of the sample were male $(n=418,68 \%)$ and one third were female $(n=195,32 \%)$. The mean age of participants at time of assessment was 38.6 
years $(S D=15.5)$. Mean period between admission and assessment was 815.9 days ( $S D=1247.4)$. The sample comprised 28 (5\%) Asian patients, 30 (5\%) Mixed ethnicity, 43 (7\%) Black, 284 (46\%) White, and 228 (37\%) with no specified ethnicity. There were 220 $(72 \%)$ patients in low secure and locked wards, and $173(28 \%)$ were in medium secure wards. Two hundred and forty-three (40\%) patients were detained under civil sections of the Mental Health Act (1983, amended 2007), 329 (54\%) under forensic sections, and 41 (7\%) were admitted informally. The sample contained 335 (55\%) patients with "Schizophrenia, schizotypal and delusional disorders" (F20-29), 229 (37\%) with "Disorders of adult personality and behaviour" (F60-69), 109 (18\%) with "Mental retardation" (F70-79), 96 (16\%) with Mental and behavioural disorders due to psychoactive substance use" (F10-19), 93 (15\%) with "Disorders of psychological development" (F80-89), 58 (10\%) had "Organic, including symptomatic, mental disorders" (F00-09), 46 (8\%) had "Mood [affective] disorders", 29 (5\%) had "Behavioural and emotional disorders with onset usually occurring in childhood and adolescence" (F90-98), 18 (3\%) had "Neurotic, stress-related and somatoform disorders" (F40-48), and 5 (1\%) had "Behavioural syndromes associated with physiological disturbances and physical factors". Percentages do not equal $100 \%$ due to comorbidity of disorders.

\section{Prevalence of verbal aggression}

In total, $341(56 \%)$ patients engaged in 1,594 incidents of any verbal aggression in the follow-up period. This comprised 199 (33\%) participants who engaged in mild verbal aggression, and 305 (50\%) who engaged in severe verbal aggression. There were 212 (13\%) incidents recorded under the least severe OAS criteria 1 for verbal aggression; 238 (15\%) incidents under criteria 2; 950 (60\%) incidents under criteria 3; and 194 (12\%) incidents under criteria 4. Significantly more males engaged in severe verbal aggression $\left(x^{2}[1\right.$, $N=380]=22.22, p<.001)$, and any verbal aggression $\left(x^{2}[1, N=432]=14.01, p<.001\right)$, than 
females. There were no significant differences in mild verbal aggression for males and females $\left(x^{2}[1, N=258]=.972, p=.324\right)$. Those under the age of 40 engaged in significantly more severe $\left(x^{2}[1, N=380]=11.43, p<.001\right\}$ and any $\left(x^{2}[1, N=432]=8.25, p<.004\right)$ verbal aggression. There were no significant differences in engagement in mild verbal aggression between those over and under the age of $40\left(x^{2}[1, N=258]=.003, p=.960\right)$.

\section{HCR-20 scores and risk estimates}

Total HCR-20, C5, and R5 scores were significantly higher for those who had engaged in mild, severe, and any verbal aggression, than for those who had not engaged in verbal aggression. H10 scores were only significantly higher in those who engaged in any verbal aggression (see Table 1).

\section{[INSERT TABLE 1 ABOUT HERE]}

There were 103 (17\%) participants assigned to low risk by the SJ, $200(33 \%)$ moderate risk, and 114 (19\%) high risk. There were 196 (32\%) participants with missing risk estimate information. A Pearson's Chi-Squared test revealed that prevalence of mild verbal aggression was highest in the HCR-20 high risk group ( $n=47,41 \%)$, and lowest in the low risk group $(n=21,20 \%) ; x^{2}(2, N=417)=11.55, p=.003$. Severe verbal aggression was most prevalent, again, in the high risk group $(n=64,56 \%)$, and lowest in the low risk group $(n=35$, $34 \%) ; x^{2}(2, N=417)=13.55, p=.001$. Prevalence of any verbal aggression was also highest in the high risk group $(n=71,62 \%)$, and lowest in the low risk group $(n=42,41 \%) ; x^{2}(2, N=417)$ $=13.16, p=.001$. Examination of the standardised residuals revealed that those in the low risk group were significantly less likely to engage in verbal aggression, compared with those rated as moderate or high risk; there were no differences in engagement between the latter two groups. 
A one-way ANOVA revealed no significant differences in H10 scores for each risk estimate; however, significant differences were found in C5, R5, and HCR-20 total scores between each risk estimate. A post hoc Tukey test revealed that C5 ( $M=5.88, S D=2.53), \mathrm{R} 5$ $(M=5.96, S D=2.52)$, and total HCR-20 $(M=25.38, S D=6.77)$ scores for the low risk group were significantly lower than scores for the moderate $(p<.001)$ and high risk $(p<.001)$ groups. No difference was found between scores for the moderate and high risk groups (see Table 2).

\section{[INSERT TABLE 2 ABOUT HERE]}

Overlap between physical and verbal aggression

A Pearson's Chi-Squared test revealed a significant overlap between physical aggression towards other people and mild verbal aggression $\left(x^{2}[1, N=199]=101.80, p<.001\right)$, severe verbal aggression $\left.x^{2}[1, N=305]=100.80, p<.001\right)$, and any verbal aggression $\left(x^{2}[1\right.$, $N=341]=109.77, p<.001)$.

\section{Predictive validity of the HCR-20}

ROC analyses revealed that none of the HCR-20 subscales had AUC values that reached the threshold for large effect size (>.75). The C5 scale produced the largest AUC values for all outcomes (.68-.69), and was significantly predictive of all categories of verbal aggression $(p<.001)$. The H10 scale had the lowest effect sizes for all outcomes and was only significantly predictive of any verbal aggression. The total HCR-20 scale and SJ were significantly predictive of all categories of verbal aggression, though effect sizes were not large (see Table 3).

Item-outcome analysis

ROC analyses, given in Table 3, revealed that none of the Historical items significantly predicted any verbal aggression. All C5 and R5 items were significant predictors 
of all categories of verbal aggression. Item C5:4 (impulsivity) had the largest effect size for mild verbal aggression and any verbal aggression; item C5:2 (negative attitudes) had the largest effect size for severe verbal aggression. Among the R5 items, Item R5:4 (noncompliance with remediation attempts) had the largest effect size for all outcomes.

\section{[INSERT TABLE 3 ABOUT HERE]}

\section{Discussion}

This study has used the largest inpatient sample in the world literature to date to examine the prevalence and severity of verbal aggression in a mental health setting and investigate its predictability using the HCR-20. Because we were also able to collate information from routinely conducted risk assessment prior to outcomes data collection we have amassed a powerful data set that has allowed us to examine whether a range of static historical and dynamic clinical and risk-related factors, predict verbal aggression of varying severity. We found that the HCR-20 was a significant predictor of all three verbal aggression categories suggesting that, in totality, items assessed on the scale represent a multivariate risk factor for this behaviour. However, this study design does not allow us to infer causality, and effect sizes were not large for any of the subscales; this suggests that there may be other important predictors of verbal aggression that are not captured by the HCR-20. The Clinical scale scores produced the largest effect sizes suggesting that current clinical functioning is the most accurate predictor of verbal aggression. In contrast, the Historical scale scores had the smallest effect sizes for all three forms of verbal aggression, and were significantly predictive only when the outcome categories were combined into the any verbal aggression category. This implies that the relatively static Historical items cannot accurately differentiate between those who do and do not engage in verbal aggression. This is consistent with previous research which has found the Historical scale to be a poor predictor of inpatient 
physical aggression (O’Shea, Picchioni, et al., 2014b), probably because most patients in secure mental health care share a high frequency of the scale's characteristics (Belfrage et al., 2000).

The HCR-20 summary clinical judgements were significantly predictive of mild, severe, and any verbal aggression, though effect sizes were smaller than that of the HCR-20 score. This is perhaps unsurprising given that the SJ refers to the raters' judgement of the likelihood that future violence will cause serious physical harm, and hence does not really apply to verbal aggression. As a result, the current findings preclude any conclusions regarding clinicians' ability to predict the likelihood of verbal aggression in a SJ, but suggest that those deemed at risk of causing serious physical harm by clinicians are at increased risk of also being verbally aggressive.

\section{Item-outcome analysis}

An important aspect of this study was the ability to examine the relationship between individual HCR-20 items and verbal aggression since this might identify specific singlevariable risk factors, and might suggest the most likely targets for effective intervention. Analysis revealed that items C5:2 (negative attitudes), C5:4 (impulsivity), and R5:4 (noncompliance with remediation attempts) were the most potent predictors of inpatient verbal aggression. This suggests that interventions aimed at reducing negative attitudes, impulsivity, and noncompliance may reduce the risk of verbal aggression. It is striking that these areas are so compatible with the cornerstones of mental health nursing practice, namely unconditional positive regard, emotional regulation, and the effective implementation of rules and boundaries (Bowers et al., 2008; Brennan et al., 2006; Flood et al., 2006). This suggests that mental health nurses are ideally placed to develop and implement effective interventions to reduce verbal aggression and to ensure it does not escalate into a more severe form of 
physical aggression. The HCR-20 Violence Risk Management Companion Guide (Douglas et al., 2001) suggests a cognitive-behaviour modification approach to changing negative and antisocial attitudes. The aim here is to change thinking and beliefs held towards others, and teach patients to recognise risky situations and know how to respond in a prosocial way (Müller-Isberner, 2001); this may be supplemented by modelling of positive regard.

Furthermore, the guide suggests that impulsivity could be managed using motivational interviewing techniques and Dialectical Behavioural Therapy; again, consistent modelling of appropriate emotional regulation should supplement this. Lastly, the use of education and motivational enhancement techniques has been suggested as methods for reducing noncompliance; mental health nurses are ideally placed to ensure the sensitive application of appropriate boundaries to promote compliance. Future research should aim to verify if any of these methods are effective in reducing verbal aggression.

\section{Base rates of verbal aggression}

Half of the sample engaged in severe verbal aggression and over half engaged in any verbal aggression. Over 1,500 incidents of verbal aggression were recorded over a threemonth follow-up period. These results are incongruent with those of Foster et al. (2007), who found a much lower incident rate of verbal aggression; 112 incidents over a 44 week period. However, closer inspection of the methodology reveals that this may be due to their measurement of only verbal threats. In contrast, Desmarais et al. (2012) measured all severities of verbal aggression and yielded similar results to ours. Though base rates of mild verbal aggression, were higher (72\%), rates of severe (56\%) and any (53\%) aggression were consistent with current findings, suggesting that measuring all forms of verbal aggression yields higher rates than measuring only one. Conversely, Stewart and Bowers (2013) reported that $263(50 \%)$ patients engaged in 1,365 incidents of verbal aggression over a 2-week period, a higher incident rate than current findings. This may be partly explained by the fact 
that the researchers recorded incidents in the first 2 weeks after admission; research has found that most aggressive incidents are displayed in the period immediately after admission (Barlow et al., 2000; Grassi et al., 2001; Holdsworth et al., 1999). However, despite the difference in event frequency, the proportion of patients engaging in verbal aggression was similar in both studies. This may be due to our method of only counting the most severe element of verbal aggression in an episode whereas Stewart and Bowers (2013), who were interested in describing the nature of verbal aggression rather than predicting its occurrence, counted each term used in an episode of the behaviour.

Research has found an under-reporting of verbal aggression (Arboleda-Florez et al., 1994). This is supported by a literature review looking at prevalence of verbal aggression against nurses; McLaughlin et al. (2009) found that the majority of nurses reported verbal aggression to be so common that it was just 'part of the job' (p. 735). This suggests that constant exposure to verbal aggression may lead to its underreporting due to the behaviour being construed as the norm. Alternatively, differences in reported base rates between studies may be due to the variation in research methods and definitions of verbal aggression used by staff recording the incidents (McLaughlin et al., 2009). A higher rate of severe verbal aggression was found in males than in females; however, there were no gender difference in mild verbal aggression. Previous research has produced conflicting results; Serper et al. (2005) reported higher verbal aggression rates among females, whereas Stone et al. (2011) found that swearing was more frequent in the male population. A possible explanation for this finding could be that verbally aggressive acts by females may be interpreted as less severe than incidents seen in males, as staff may anticipate and recognise aggression more readily in males (Hiday et al., 1998).

Higher rates of severe verbal aggression and any verbal aggression were found in those under the age of 40 , compared with those over the age of 40 . There were no age 
differences found in the rates of mild verbal aggression. These findings are congruent with previous research that has found verbal aggression was more likely to occur in younger patients (Stewart \& Bowers, 2013). Furthermore, Barlow et al. (2000) identified high risk patients as those under the age of 32 , suggesting that younger patients are more prone to aggression.

\section{Limitations}

One factor that may have compromised the predictive validity of the HCR-20 is that the period of follow-up after assessment was three months. O'Shea et al. (2013) have found that using a longer time frame, e.g. six months, may increase the predictive ability of the HCR-20, as it allows for greater opportunity for events to occur and increases the likelihood of their detection. However, we selected a three-month period in order to maximise the number of patients who could be followed-up over a uniform time period rather than including various follow-up periods. This strategy strengthened the study's ability to provide results that can be confidently applied to a specific time period. While it potentially lessens generalisability by reducing the overall sample size by excluding patients who did not stay for three months, the mean length of stay for patients in St. Andrew's is 2 years, and it was therefore expected that the proportion of patients excluded would be low. If the alternative follow-up period of six months was used, a higher attrition rate than the $4 \%$ in this study would have been experienced.

A further limitation is that the intervention techniques used on the wards may have affected the predictive efficacy of the HCR-20, as actual verbal aggression incidents may not have taken place due to successful intervention. However, this is impossible to overcome, as it is necessary for staff to try to de-escalate situations when they recognise markers of 
agitation. Therefore, due to the design of this study, the predictive validity of the HCR-20 may underestimate its true abilities as a result of lower incidence rates.

This study focused on patient related risk factors for verbal aggression, as these factors are the focus of the HCR-20. However, our results strongly support the proposition that there are many other variables, unrelated to patients' gender, age, or diagnosis, that contribute to their engagement in aggressive behaviour. Hallett et al. (2014) report both staff and environmental factors influence patient engagement or non-engagement in aggressive behaviours. Staff factors such as de-escalation skills (Dickens et al., 2013), and the style and quality of communication with patients are reported by both mental health staff (Bond \& Brimblecombe, 2003) and patients (Kumar et al., 2001) to have considerable effects on whether patients will engage in aggression. Negative staff-related attributes, for example staff appearing to talk among themselves or being unavailable, are also widely held to underlie some patient aggression (Meehan et al., 2006). Furthermore, environmental factors such as staffing levels and skills mix on wards have been perceived by staff and patients to affect the frequency and severity of aggressive incidents (Chaplin et al., 2006). Therefore, we strongly endorse that the effective management of verbal aggression in secure mental health care requires full consideration of staff and environmental factors, in addition to patient factors.

A further limitation of this study is that $32 \%$ of the sample had missing risk estimates, which may have resulted in the smaller effect sizes found for this predictor. Additionally, $37 \%$ of the sample had no recorded ethnicity. Further, this study did not examine predictive validity of the HCR-20 in different populations and demographic factors were not controlled for. This, then, could be a limitation as previous research suggests that predictive validity is stronger in certain patient populations, such as those with schizophrenia (O'Shea, Picchioni, et al. (2014b). Controlling for demographic factors to measure predictive validity in different populations could therefore have yielded larger effect sizes specific to that population. 


\section{Implications and further considerations}

Results indicate that the HCR-20 is somewhat predictive of verbal aggression, though effect sizes are not large. Item-outcome analysis has demonstrated that impulsivity, negative attitudes, and non-compliance were the strongest predictors of verbal aggression; establishing whether risk management techniques in the HCR-20 companion guide (Douglas et al., 2001) aimed at targeting these factors are effective in reducing verbal aggression is yet to be investigated. These areas are fully compatible with the mental health nursing role and should be central to nursing practice in these settings.

Future research should investigate whether particular dynamic variables are better predictors of verbal aggression in different categories of patients. For example, O'Shea, Picchioni, et al. (2014b) found that the HCR-20 was a better predictor of violence in those with schizophrenia, personality disorder, and women; examining the efficacy of each HCR20 item in predicting verbal aggression for different patient groups could enable more suitable interventions to be applied. Further, it may be desirable to test the feasibility and accuracy of a summary judgement for verbal aggression which would have the potential to inform treatment and safety planning. 


\section{References}

Arboleda-Florez, J., Crisanti, A., Rose, S., \& Holley, H. (1994). Measuring aggression on psychiatric inpatient units: development and testing of the Calgary General Hospital Aggression Scale. International Journal of Offender Therapy and Comparative Criminology, 38(3), 183-204.

Arnetz, J. E., \& Arnetz, B. B. (2001). Violence towards health care staff and possible effects on the quality of patient care. Social Science \& Medicine, 52(3), 417-427.

Barlow, K., Grenyer, B., \& Ilkiw-Lavalle, O. (2000). Prevalence and precipitants of aggression in psychiatric inpatient units. Australian and New Zealand Journal of Psychiatry, 34(6), 967-974.

Belfrage, H., Fransson, R., \& Strand, S. (2000). Prediction of violence using the HCR-20: a prospective study in two maximum-security correctional institutions. The Journal of Forensic Psychiatry, 11(1), 167-175.

Bond, K., \& Brimblecombe, N. (2003). Staff views on how violent incidents take place. Mental Health Nursing-London-, 23(6), 10-13.

Bowers, L., Flood, C., Brennan, G., \& Allan, T. (2008). A replication study of the City nurse intervention: reducing conflict and containment on three acute psychiatric wards. Journal of psychiatric and mental health nursing, 15(9), 737-742.

Brennan, G., Flood, C., \& Bowers, L. (2006). Constraints and blocks to change and improvement on acute psychiatric wards-lessons from the City Nurses project. Journal of psychiatric and mental health nursing, 13(5), 475-482.

Chaplin, R., McGeorge, M., \& Lelliott, P. (2006). The National Audit of Violence: in-patient care for adults of working age. Psychiatric Bulletin, 30(12), 444-446.

Dernevik, M., Grann, M., \& Johansson, S. (2002). Violent behaviour in forensic psychiatric patients: risk assessment and different risk-management levels using the HCR-20. Psychology, Crime \& Law, 8(1), 93-111.

Desmarais, S. L., Nicholls, T. L., Wilson, C. M., \& Brink, J. (2012). Using dynamic risk and protective factors to predict inpatient aggression: reliability and validity of START assessments. Psychological assessment, 24(3), 685.

Dickens, G., Piccirillo, M., \& Alderman, N. (2013). Causes and management of aggression and violence in a forensic mental health service: perspectives of nurses and patients. International journal of mental health nursing, 22(6), 532-544.

Douglas, K. S., Hart, S., Webster, C., \& Belfrage, H. (2013). HCR-20 version 3: assessing risk for violence. Burnaby, BC, Canada: Mental Health, Law and Policy Institute, Simon Fraser University.

Douglas, K. S., Webster, C., \& Hart, S. (2001). HCR-20: Violence risk management companion guide. Mental Health Law \& Policy Faculty Publications.

Doyle, M., \& Dolan, M. (2002). Violence risk assessment: combining actuarial and clinical information to structure clinical judgements for the formulation and management of risk. Journal of Psychiatric and Mental Health Nursing, 9(6), 649-657.

Duxbury, J. (2002). An evaluation of staff and patient views of and strategies employed to manage inpatient aggression and violence on one mental health unit: a pluralistic design. Journal of psychiatric and mental health nursing, 9(3), 325-337.

Flannery Jr., R. B., Hanson, M. A., \& Penk, W. E. (1995). Patients' threats expanded definition of assault. General Hospital Psychiatry, 17(6), 451-453.

Flood, C., Brennan, G., Bowers, L., Hamilton, B., Lipang, M., \& Oladapo, P. (2006). Reflections on the process of change on acute psychiatric wards during the City Nurse Project. Journal of psychiatric and mental health nursing, 13(3), 260-268. 
Foster, C., Bowers, L., \& Nijman, H. (2007). Aggressive behaviour on acute psychiatric wards: prevalence, severity and management. Journal of advanced nursing, 58(2), 140-149. doi: 10.1111/j.1365-2648.2007.04169.x

Grassi, L., Peron, L., Marangoni, C., Zanchi, P., \& Vanni, A. (2001). Characteristics of violent behaviour in acute psychiatric in-patients: a 5-year Italian study. Acta Psychiatrica Scandinavica, 104(4), 273-279.

Guy, L. S., Douglas, K. S., \& Hendry, M. C. (2010). The role of psychopathic personality disorder in violence risk assessments using the HCR-20. Journal of personality disorders, 24(5), 551-580.

Hallett, N., Huber, J. W., \& Dickens, G. L. (2014). Violence prevention in inpatient psychiatric settings: systematic review of studies about the perceptions of care staff and patients. Aggression and Violent Behavior, 19(5), 502-514.

Hare, R. (1991). The Hare psychopathy checklist-revised. Multi-Health Systems, Toronto.

Hiday, V., Swartz, M., Swanson, J., Borum, R., \& Wagner, H. (1998). Male-female differences in the setting and construction of violence among people with severe mental illness. Social Psychiatry and Psychiatric Epidemiology, 33(1), S68-S74.

Holdsworth, Collis, \& Allott. (1999). The development and evaluation of a brief risk screening instrument for the psychiatric inpatient setting. Journal of psychiatric and mental health nursing, 6(1), 43-52. doi: 10.1046/j.1365-2850.1999.00179.x

Kay, S., Wolkenfeld, F., \& Murrill, L. (1988). Profiles of aggression among psychiatric patients. I. Nature and prevalence. Journal of nervous and mental disease, 176(9), 539-546.

Kumar, S., Guite, H., \& Thornicroft, G. (2001). Service users' experience of violence within a mental health system: a study using grounded theory approach. Journal of mental Health, 10(6), 597-611.

McLaughlin, S., Gorley, L., \& Moseley, L. (2009). The prevalence of verbal aggression against nurses. British journal of nursing, 18(12), 735-739.

Meehan, T., McIntosh, W., \& Bergen, H. (2006). Aggressive behaviour in the high-secure forensic setting: the perceptions of patients. Journal of Psychiatric and Mental Health Nursing, 13(1), 19-25.

Müller-Isberner, R. (2001). Changing attitudes: effecting positive and lasting changes in HCR-20: violence risk management companion guide. Mental Health Law \& Policy Faculty Publications., pp. 85-91.

Needham, I., Abderhalden, C., Halfens, R. J., Fischer, J. E., \& Dassen, T. (2005). Non-somatic effects of patient aggression on nurses: a systematic review. Journal of advanced nursing, 49(3), 283-296.

O’Connell, B., Young, J., Brooks, J., Hutchings, J., \& Lofthouse, J. (2000). Nurses' perceptions of the nature and frequency of aggression in general ward settings and high dependency areas. Journal of clinical Nursing, 9(4), 602-610.

O’Shea, L. E., Picchioni, M. M., Mason, F. L., Sugarman, P. A., \& Dickens, G. L. (2014a). Predictive validity of the HCR-20 for inpatient self-harm. Comprehensive Psychiatry.

O’Shea, L. E., Picchioni, M. M., Mason, F. L., Sugarman, P. A., \& Dickens, G. L. (2014b). Differential predictive validity of HCR-20 for inpatient aggression. Psychiatry Research. doi: 10.1016/j.psychres.2014.07.080

O’Shea, L. E., Thaker, D. K., Picchioni, M. M., Mason, F. L., Knight, C., \& Dickens, G. L. (2014). Predictive validity of the HCR-20 for violent and non-violent sexual behaviour in a secure mental health service. Manuscript submitted for publication.

O'Shea, L. E., Mitchell, A. E., Picchioni, M. M., \& Dickens, G. L. (2013). Moderators of the predictive efficacy of the Historical, Clinical and Risk Management-20 for aggression 
in psychiatric facilities: systematic review and meta-analysis. Aggression and Violent Behavior, 18(2), 255-270.

Rippon, T. J. (2000). Aggression and violence in health care professions. Journal of advanced nursing, 31(2), 452-460.

Serper, M. R., Goldberg, B. R., Herman, K. G., Richarme, D., Chou, J., Dill, C. A., \& Cancro, R. (2005). Predictors of aggression on the psychiatric inpatient service. Comprehensive Psychiatry, 46(2), 121-127.

SPSS, I. (2009). PASW Statistics for Windows, Version 18.0. Chicago: SPSS Inc. .

Stewart, D., \& Bowers, L. (2013). Inpatient verbal aggression: content, targets and patient characteristics. Journal of psychiatric and mental health nursing, 20(3), 236-243.

Stone, T. E., McMillan, M., \& Hazelton, M. (2010). Swearing: Its prevalence in healthcare settings and impact on nursing practice. Journal of psychiatric and mental health nursing, 17(6), 528-534.

Stone, T., McMillan, M., Hazelton, M., \& Clayton, E. H. (2011). Wounding words: swearing and verbal aggression in an inpatient setting. Perspectives in psychiatric care, 47(4), 194-203.

Webster, C. D., Douglas, K. S., Eaves, D., \& Hart, S. D. (1997). Assessing risk of violence to others. Impulsivity: Theory, assessment, and treatment, 251-277.

Werner, P., Yesavage, J., Becker, J., Brunsting, D., \& Isaacs, J. (1983). Hostile words and assaultive behavior on an acute inpatient psychiatric unit. The Journal of nervous and mental disease, 171(6), 385-387.

World Health Organization. (1992). The ICD-10 classification of mental and behavioural disorders: clinical descriptions and diagnostic guidelines.

Yudofsky, S., Silver, J., Jackson, W., Endicott, J., \& Williams, D. (1986). The Overt Aggression Scale for the objective rating of verbal and physical aggression. The American journal of psychiatry, 143(1), 35-39. 
Table 1: Differences in HCR-20 scores for mild, severe, and any verbal aggression.

\begin{tabular}{|c|c|c|c|c|c|c|c|c|c|}
\hline \multirow{2}{*}{$\begin{array}{l}\text { Mean HCR-20 } \\
\text { scores }(S D)\end{array}$} & \multicolumn{3}{|c|}{ Mild Verbal Aggression } & \multicolumn{3}{|c|}{ Severe Verbal Aggression } & \multicolumn{3}{|c|}{ Any Verbal Aggression } \\
\hline & Yes & No & Test & Yes & No & Test & Yes & No & Test \\
\hline H10 & $3.96(2.99)$ & $3.71(2.03)$ & $t(611)=-.98, p=.326$ & $3.99(2.92)$ & $3.59(3.10)$ & $\mathrm{t}(611)=-1.61, p=.108$ & $4.02(3.09)$ & $3.50(2.93)$ & $t(611)=-2.13, p=.033$ \\
\hline C5 & $.80(1.93)$ & $.34(2.36)$ & $t(469)=-8.12, p<.001$ & $.52(2.09)$ & $.11(2.35)$ & $t(604)=-7.87, p<.001$ & $.49(2.09)$ & $.96(2.34)$ & $t(611)=-8.56, p<.001$ \\
\hline R5 & $.53(2.29)$ & $.38(2.48)$ & $t(611)=-5.50, p<.001$ & $.41(2.27)$ & $.10(2.52)$ & $t(611)=-6.76, p<.001$ & $.34(2.29)$ & $.01(2.51)$ & $t(611)=-6.84, p<.001$ \\
\hline Total & $9.30(4.81)$ & $6.41(5.66)$ & $t(453)=-6.57, p<.001$ & $8.93(4.95)$ & $5.78(5.70)$ & $t(601)=-7.31, p<.001$ & $8.86(4.94)$ & $5.44(5.72)$ & $t(537)=-7.81, p<.001$ \\
\hline
\end{tabular}

H10, Historical subscale of the HCR-20; C5, Clinical subscale of the HCR-20; R5, Risk-management subscale of the HCR-20 
Table 2: HCR-20 scores for low, moderate, and high risk estimates.

\begin{tabular}{lllll}
\hline Mean HCR-20 scores $(S D)$ & Low & Moderate & High & Test \\
\hline H10 & $13.54(3.38)$ & $14.35(2.38)$ & $14.13(3.21)$ & $F(2,352)=2.23, p=.109$ \\
C5 & $5.88(2.53)$ & $7.11(2.23)$ & $7.17(2.14)$ & $F(2,352)=10.23, p<.001$ \\
R5 & $5.96(2.52)$ & $7.37(2.22)$ & $7.89(2.25)$ & $F(2,352)=17.95, p<.001$ \\
HCR-20 Total & $25.38(6.77)$ & $28.83(4.83)$ & $29.19(5.61)$ & $F(2,352)=13.1, p<.001$
\end{tabular}

H10, Historical subscale of the HCR-20; C5, Clinical subscale of the HCR-20; R5, Risk-management subscale of the HCR-20 
Table 3: Validity of individual items, HCR-20 and SJ in predicting verbal aggression.

\begin{tabular}{|c|c|c|c|c|c|c|c|c|c|}
\hline \multirow{2}{*}{$\begin{array}{l}\text { HCR-20 } \\
\text { items }\end{array}$} & \multicolumn{3}{|c|}{ Mild Verbal Aggression } & \multicolumn{3}{|c|}{ Severe Verbal Aggression } & \multicolumn{3}{|c|}{ Any Verbal Aggression } \\
\hline & $\overline{\text { AUC }}$ & 99\% CI & $p$-value & $\overline{\text { AUC }}$ & 99\% CI & $p$-value & $\overline{\text { AUC }}$ & $99 \% \mathrm{CI}$ & $p$-value \\
\hline HCR-20 & .650 & {$[.589$} & .000 & .668 & {$[.612$,} & .000 & .681 & {$[.625$,} & .000 \\
\hline Total & & $.710]$ & & & $.724]$ & & & .737] & \\
\hline \multirow[t]{2}{*}{ H1 } & .525 & {$[.462$,} & .318 & .526 & {$[.466$,} & .269 & .528 & {$[.467$,} & .235 \\
\hline & & $.588]$ & & & $.586]$ & & & $.589]$ & \\
\hline \multirow[t]{2}{*}{$\mathbf{H 2}$} & .508 & {$[.444$,} & .737 & .534 & {$[.473$,} & .150 & .540 & {$[.480$} & .088 \\
\hline & & $.537]$ & & & $.594]$ & & & $.601]$ & \\
\hline \multirow[t]{2}{*}{ H3 } & .520 & {$[.455$} & .434 & .490 & {$[.428$,} & .682 & .499 & {$[.436$,} & .953 \\
\hline & & $.585]$ & & & $.552]$ & & & $.561]$ & \\
\hline \multirow[t]{2}{*}{ H4 } & .545 & {$[.478$,} & .083 & .532 & {$[.470$,} & .187 & .548 & {$[.486$} & .048 \\
\hline & & $.612]$ & & & $.595]$ & & & $.611]$ & \\
\hline \multirow[t]{2}{*}{ H5 } & .481 & [.416, & .446 & .509 & {$[.448$,} & .710 & .507 & {$[.447$,} & .754 \\
\hline & & $.546]$ & & & $.569]$ & & & $.568]$ & \\
\hline \multirow[t]{2}{*}{ H6 } & .535 & {$[.473$,} & .160 & .521 & {$[.460$,} & .379 & .527 & {$[.466$,} & .258 \\
\hline & & $.598]$ & & & $.581]$ & & & $.587]$ & \\
\hline \multirow[t]{2}{*}{ H8 } & .481 & {$[.415$,} & .448 & .504 & {$[.444$,} & .854 & .511 & {$[.450$,} & .640 \\
\hline & & .547] & & & $.565]$ & & & $.572]$ & \\
\hline \multirow[t]{2}{*}{ H9 } & .491 & {$[.423$,} & .723 & .515 & {$[.452$,} & .467 & .506 & {$[.442$,} & .805 \\
\hline & & $.558]$ & & & $.579]$ & & & $.571]$ & \\
\hline \multirow[t]{2}{*}{ H10 } & .494 & {$[.429$,} & .445 & .504 & {$[.444$,} & .870 & .509 & {$[.449$,} & .695 \\
\hline & & $.559]$ & & & $.564]$ & & & $.570]$ & \\
\hline \multirow[t]{2}{*}{ Total } & .522 & {$[.457$} & .383 & .538 & {$[.478$,} & .106 & .551 & {$[.491$} & .030 \\
\hline & & $.586]$ & & & $.598]$ & & & $.611]$ & \\
\hline \multirow[t]{2}{*}{ C1 } & .573 & {$[.511$,} & .003 & .572 & {$[.512$,} & .002 & .562 & {$[.502$,} & .008 \\
\hline & & .635] & & & .631] & & & .623] & \\
\hline \multirow[t]{2}{*}{ C2 } & .635 & {$[.574$,} & .000 & .650 & {$[.592$,} & .000 & .658 & {$[.601$,} & .000 \\
\hline & & .695] & & & .707] & & & $.716]$ & \\
\hline C3 & .557 & {$[.494$,} & .024 & .564 & {$[.504$,} & .006 & .566 & {$[.506$} & .005 \\
\hline
\end{tabular}




\begin{tabular}{|c|c|c|c|c|c|c|c|c|c|}
\hline & & .619] & & & $.624]$ & & & .627] & \\
\hline \multirow[t]{2}{*}{$\mathrm{C} 4$} & .666 & {$[.608$,} & .000 & .640 & {$[.582$,} & .000 & .672 & [.615, & .000 \\
\hline & & $.724]$ & & & .698] & & & $.729]$ & \\
\hline \multirow[t]{2}{*}{ C5 } & .581 & {$[.518$,} & .001 & .579 & {$[.519$,} & .001. & .584 & {$[.524$,} & .000 \\
\hline & & .644] & & & .638] & & & .644] & \\
\hline \multirow[t]{2}{*}{ Total } & .683 & {$[.625$,} & .000 & .679 & {$[.624$,} & .000 & 693 & [.639, & .000 \\
\hline & & $.742]$ & & & $.735]$ & & & $.748]$ & \\
\hline \multirow[t]{2}{*}{ R1 } & .586 & {$[.523$,} & .001 & .600 & {$[.541$,} & .000 & .605 & {$[.546$,} & .000 \\
\hline & & .649] & & & .659] & & & .665] & \\
\hline \multirow[t]{2}{*}{$\mathbf{R} 2$} & .573 & {$[.510$,} & .004 & .599 & {$[.540$,} & .000 & .601 & {$[.542$,} & .000 \\
\hline & & .635] & & & .657] & & & .661] & \\
\hline \multirow[t]{2}{*}{$\mathbf{R 3}$} & .562 & {$[.498$,} & .013 & .552 & {$[.491$,} & .028 & .556 & [.495, & .018 \\
\hline & & .625] & & & .612] & & & .616] & \\
\hline \multirow[t]{2}{*}{ R4 } & .615 & {$[.552$,} & .000 & .634 & {$[.576$,} & .000 & .626 & {$[.568$,} & .000 \\
\hline & & .678] & & & .692] & & & .685] & \\
\hline \multirow[t]{2}{*}{ R5 } & .600 & {$[.540$,} & .000 & .610 & {$[.551$,} & .000 & .612 & {$[.552$,} & .000 \\
\hline & & $.661]$ & & & .669] & & & .672] & \\
\hline \multirow[t]{2}{*}{ Total } & .636 & {$[.575$,} & .000 & .650 & {$[.594$,} & .000 & .654 & [.596, & .000 \\
\hline & & .697] & & & .707] & & & $.712]$ & \\
\hline \multirow[t]{2}{*}{ SJ } & .588 & {$[.514$,} & .003 & .583 & {$[.511$,} & .003 & .582 & {$[.509$,} & .004 \\
\hline & & .663] & & & .655] & & & .655] & \\
\hline
\end{tabular}

H, Historical items in the HCR-20; C, Clinical items in the HCR-20; R, Risk-management items in the HCR-20; SJ, summary judgement; AUC, area under receiver operating characteristic curve; CI, confidence interval 